\title{
INTERACTION BETWEEN PARAMETRIC AND FORCED OSCILLATIONS IN A QUASI-LINEAR OSCILLATING SYSTEM
}

\author{
Tran Kim Chi - NGuYen Van Dinh \\ Institute of Mechanics, Hanoi Vietnam
}

In [1], a quasi-linear oscillating system with time-dependent quadratic non-linearity subjected to external excitation has been considered. It has been shown that there exists a cert ain interaction between parametric and forced oscillations due to the quadratic nonlinearity and the external excitation respectively. The fundamental resonance in the case of dephase $\pi$ between two excitations has been analyzed and two forms of the resonance curve have been obtained.

In the present paper the general case with arbitrary dephase between two excitations will be examined. Critical singular points [2] will be used to classify different forms of the resonance curve.

1. System under consideration. Ordinary and critical stationary cscillations

Let us consider a quasi-linear oscillating system described by the differential equation:

$$
\ddot{x}+\omega^{2} x=\varepsilon\left\{-h \dot{x}+\Delta x-\gamma x^{3}+2 p x^{2} \cos \omega t+e \cos (\omega t+\sigma)\right\},
$$

where : $x$ is an oscillatory variable; overdots denote the differentiation with respect to time $t ; \varepsilon>0$ is a small parameter; $h \geq 0$ is the damping coefficient; $\gamma$ is the cubic non-linearity coefficient; $e>0$, $2 p>0$ and $\omega$ are intensities and common frequency of the external and parametric excitations, respectively; $\sigma(0 \leq \sigma<2 \pi)$ is the dephase between two excitations; $\varepsilon \Delta=\omega^{2}-1$ is the detuning parameter ( 1 - own frequency).

Introducing slowly varying variables $(a, \theta)$ - amplitude and phase of the oscillations - by mean of the formulae:

$$
x=a \cos \psi, \quad \dot{x}=-\omega a \sin \psi, \quad \psi=\omega t+\theta
$$

and using the asymptotic method, we obtain, in the first approximation, the following averaged differential equations:

$$
\begin{aligned}
\dot{a} & =-\frac{\varepsilon}{2 \omega} f=-\frac{\varepsilon}{2 \omega}\left\{h \omega a+\left(\frac{1}{2} p a^{2}+e \cos \sigma\right) \sin \theta-e \sin \sigma \cdot \cos \theta\right\}, \\
a \dot{\theta} & =-\frac{\varepsilon}{2 \omega} g=-\frac{\varepsilon}{2 \omega}\left\{-\left(\frac{3 \gamma}{4} a^{2}-\Delta\right) a+e \sin \sigma \sin \theta+\left(\frac{3}{2} p a^{2}+e \cos \sigma\right) \cos \theta\right\} .
\end{aligned}
$$

Stationary oscillations of constant amplitude and phase will be determined from the equations:

$$
f=0, \quad g=0 .
$$

By $D_{0}, D_{1}, D_{2}$ we denote the determinants:

$$
D_{0}=\left|\begin{array}{cc}
\frac{1}{2} p a^{2}+e \cos \sigma & -e \sin \sigma \\
e \sin \sigma & \frac{3}{2} p a^{2}+e \cos \sigma
\end{array}\right|,
$$




$$
D_{1}=\left|\begin{array}{cc}
-h \omega a & -e \sin \sigma \\
\left(\frac{3 \gamma}{4} a^{2}-\Delta\right) a & \frac{3}{2} p a^{2}+e \cos \sigma
\end{array}\right|, \quad D_{2}=\left|\begin{array}{cc}
\frac{1}{2} p a^{2}+e \cos \sigma & -h \omega a \\
e \sin \sigma & \left(\frac{3 \gamma}{4} a^{2}-\Delta\right) a
\end{array}\right| .
$$

In the ordinary region, where

$$
D_{0}=\left(\frac{1}{2} p a^{2}+e \cos \sigma\right)\left(\frac{3}{2} p a^{2}+e \cos \sigma\right)+(e \sin \sigma)^{2} \neq 0,
$$

from (1.4), $\sin \theta$ and $\cos \theta$ can be calculated and the ordinary part $C_{1}$ of the resonance curve $C$ is obtained

$$
W_{1}\left(\Delta, a^{2}\right)=\frac{D_{1}^{2}+D_{2}^{2}}{D_{0}^{2}}-1=0 .
$$

The critical region is characterized by the equality:

$$
D_{0}=\frac{3}{4} p^{2}\left(a^{2}\right)^{2}+2 p(e \cos \sigma) a^{2}+e^{2}=0 .
$$

Evidently, (1.8) has positive solutions $a^{2}$ if:

$$
-1 \leq \cos \sigma \leq \frac{-\sqrt{3}}{2}
$$

Thus: - if $0 \leq \sigma<\frac{5 \pi}{6}$ or $\frac{7 \pi}{6}<\sigma<2 \pi$, the critical region does not exist,

- if $\frac{5 \pi}{6} \leq \sigma \leq \frac{7 \pi}{6}$, the critical region exists and consists of two straight parallel lines:

$$
a^{2}=\frac{4 e}{3 p}\left(-\cos \sigma \pm \sqrt{\cos ^{2} \sigma-\frac{3}{4}}\right)
$$

(a dowble straight line if $\sigma=\frac{5 \pi}{6}$ or $\sigma=\frac{7 \pi}{6}$ )

To determine the critical part $C_{2}$ (of the resonance curve $C$ ) we have to solve the equations:

$$
D_{0}=0, \quad D_{1}=0, \quad D_{2}=0
$$

under the restrictions:

$$
\begin{aligned}
& \left(\frac{1}{2} p a^{2}+e \cos \sigma\right)^{2}+(e \sin \sigma)^{2} \geq h^{2} \omega^{2} a^{2} \\
& (e \sin \sigma)^{2}+\left(\frac{3}{2} p a^{2}+e \cos \sigma\right)^{2} \geq\left(\frac{3 \gamma}{4} a^{2}-\Delta\right)^{2} a^{2} .
\end{aligned}
$$

As it has been shown in [2], by rejecting those points $\left(\Delta, a^{2}\right)$ satisfying (1.11) but not (1.12), the "whole" resonance curve $C\left(C_{1}+C_{2}\right)$ can be found from the relationship:

$$
W\left(\Delta, a^{2}\right)=D_{1}^{2}+D_{2}^{2}-D_{0}^{2}=0 .
$$

By $D$ we denote the discriminant:

$$
D=\left(\frac{\partial^{2} W}{\partial \Delta \partial a^{2}}\right)^{2}-\left(\frac{\partial^{2} W}{\partial \Delta^{2}}\right)\left(\frac{\partial^{2} W}{\partial a^{2} \partial a^{2}}\right)
$$


- if $D>0, I$ is a nodal point

- if $D<0, \mathrm{I}$ is an isolated point

\section{System with dephase $\pi$}

This case has been analyzed in [1]. In this case, the system (1.11) (1.12) and the relationship (1.13) are simple:

$$
\begin{gathered}
D_{0}=\left(\frac{1}{2} p a^{2}-e\right)\left(\frac{3}{2} p a^{2}-e\right)=0 \\
D_{1}=-h \omega a\left(\frac{3}{2} p a^{2}-e\right)=0 \\
D_{2}=a\left(\frac{3 \gamma}{4} a^{2}-\Delta\right)\left(\frac{1}{2} p a^{2}-e\right)=0 \\
\left(\frac{1}{2} p a^{2}-e\right)^{2} \geq h^{2} \omega^{2} a^{2} \\
\left(\frac{3}{2} p a^{2}-e\right)^{2} \geq\left(\frac{3 \gamma}{4} a^{2}-\Delta\right)^{2} a^{2}, \\
W\left(\Delta, a^{2}\right)=h^{2} \omega^{2} a^{2}\left(\frac{3}{2} p a^{2}-e\right)^{2}+a^{2}\left(\frac{1}{2} p a^{2}-e\right)^{2}\left(\frac{3 \gamma}{4} a^{2}-\Delta\right)^{2}, \\
-\left(\frac{1}{2} p a^{2}-e\right)^{2}\left(\frac{3}{2} p a^{2}-e\right)^{2}=0 .
\end{gathered}
$$

If $h=0$ (system without damping), from (2.1) we obtain:

- a compatible point $I_{*}$ of coordinates $\left(\Delta_{i}, a_{i}^{2}\right)$ :

$$
\Delta_{i}=\frac{3 \gamma}{4} a_{i}^{2}, \quad a_{i}^{2}=\frac{2 e}{3 p}
$$

- a compatible line $J$ parallel to the abscissa axis $\Delta$ :

$$
a^{2}=\frac{2 e}{p}
$$

It is not difficult to verify that:

- I satisfies (2.2) i.e. I corresponds to critical stationary oscillations, it's a part of $C_{2}$.

- Along $J,(2.2)$ is satisfied only, if:

$$
\frac{3 \gamma}{4} a_{j}^{2}-\sqrt{2 e p} \leq \Delta \leq \frac{3 \gamma}{4} a_{j}^{2}+\sqrt{2 e p}
$$

i.e. along $J$, only segment $J_{1} J_{2}$ determined by $(2.5)(2.6)$ is representing segment - the second part of $C_{2}$.

If $h>0$ (system with damping), the compatibility conditions (2.1) admit only I as compatible point at which the trigonometrical conditions $(2.2)$ lead to the inequality:

$$
h^{2} \leq h_{c}^{2}=\frac{4 e p^{2}}{3(\gamma e+2 p)} .
$$

Thus : - if $h \leq h_{c}$, I is a representing critical point; 
- if $h>h_{c}, \mathrm{I}$ is an isolated point (and must be rejected).

The resonance curve of the system with damping consists of two branches: the upper $C^{\prime}$ and the lower $C^{\prime \prime}$ lying respectively above and under the line $J$. The upper branch $C^{\prime}$ is of "parabolic" form, the lower one $C^{\prime \prime}$ has a loop with the nodal point I. As $h$ increases, $C^{\prime}$ moves up, whereas $C^{\prime \prime}$ moves down, the loop becomes narrower then disappears.

In Fig. 1, for fixed values $\sigma=\pi, \gamma=0.1 ; e=0.06 ; p=0.2$ the resonance curve $1,2,3$ corresponds to $h=0 ; 0.005 ; 0.1$.

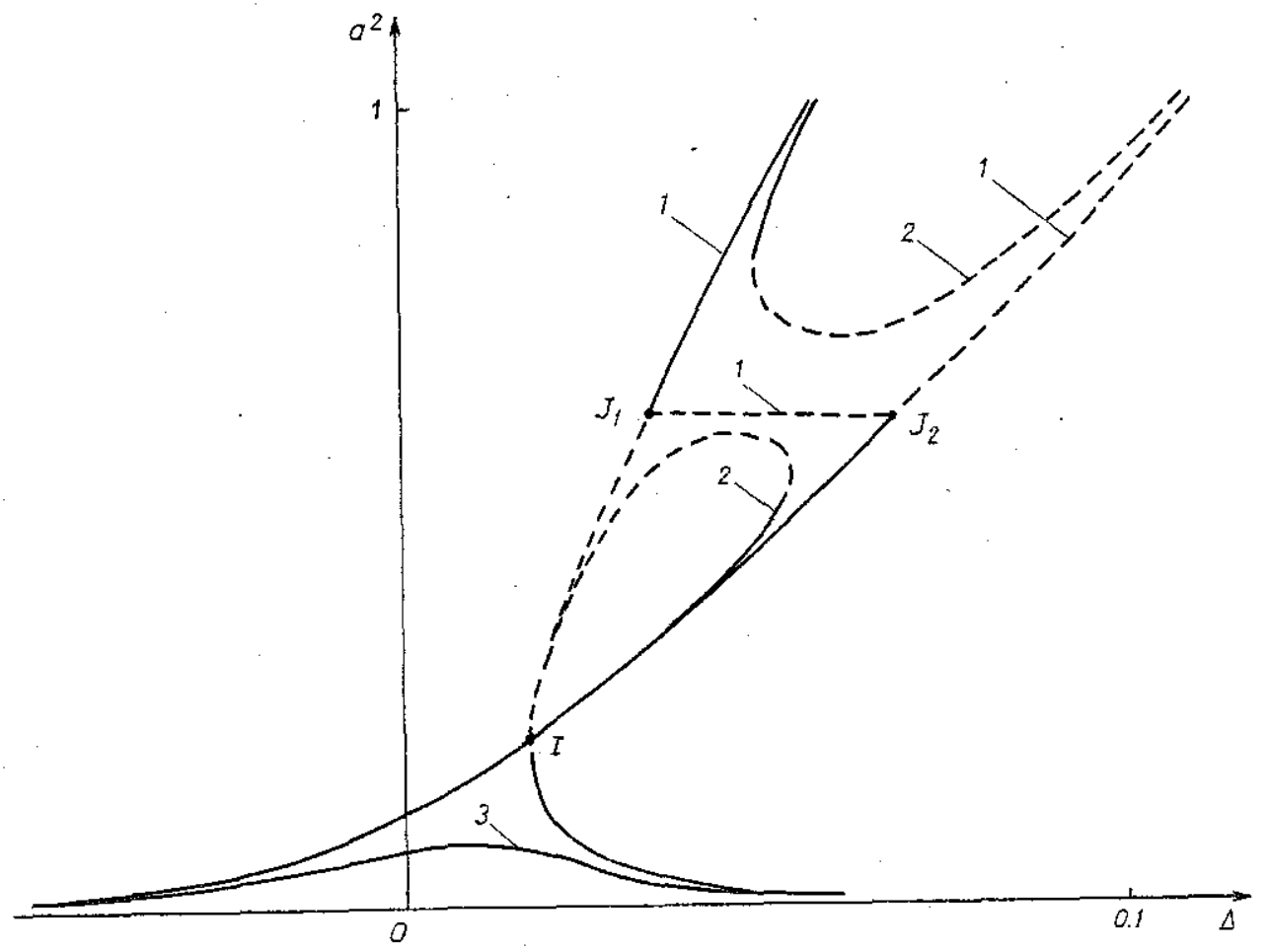

Fig. 1

3. The system with dephase $\frac{5 \pi}{6} \leq \sigma \neq \pi \leq \frac{7 \pi}{6}$

In this case, there are two compatible points I, J. Indeed, from the first equation $D_{0}=0$ of the system (1.11), we have obtained the ordinates:

$$
a_{i, j}^{2}=\frac{4 e}{3 p}\left(-\cos \sigma \pm \sqrt{\cos ^{2} \sigma-\frac{3}{4}}\right)
$$

The second equation $D_{1}=0$ can be written as:

$$
D_{1}=(e \sin \sigma) \omega^{2}+\left(\frac{3}{2} p a_{i j}^{2}+e \cos \sigma\right) h \omega-\left(\frac{3 \gamma}{4} a_{i j}^{2}+1\right) e \sin \sigma=0 .
$$

The positive roots are:

$\omega_{i, j}=\sqrt{1+\Delta_{i, j}}=\frac{-\left(\frac{3}{2} p a_{i j}^{2}+e \cos \sigma\right) h+\sqrt{\left(\frac{3}{2} p a_{i j}^{2}+e \cos \sigma\right)^{2} h^{2}+4\left(\frac{3 \gamma}{4} a_{i j}^{2}+1\right) e^{2} \sin ^{2} \sigma}}{2 e \sin \sigma}$ 
If $h=0$, we have:

$$
\omega_{i, j}=\frac{3 \gamma}{4} a_{i, j}^{2}+1 \quad \text { or } \quad \frac{3 \gamma}{4} a_{i j}^{2}=\Delta_{i, j}
$$

i.e. $I$ and $J$ lie in the skeleton curve $\frac{3 \gamma}{4} a^{2}=\Delta$.

Evidently, the trigonometrical conditions (1.12) are satisfied. Thus, for the system without damping, if $\frac{5 \pi}{6} \leq \sigma \neq \pi \leq \frac{7 \pi}{6}$, the resonance curve admits two critical points $\mathrm{I}$, $\mathrm{J}$ lying in the skeleton curve (a double critical point if $\sigma=\frac{5 \pi}{6}$ or $\sigma=\frac{7 \pi}{6}$ ); these two points are of nodal type.

Increasing $h, \mathrm{I}, \mathrm{J}$ move along two critical lines (1.10) respectively.

Differentiating (3.3) with respect to $h$, we obtain:

$$
\frac{d \omega_{i j}}{d h}=\frac{\left(\frac{3}{2} p a_{i j}^{2}+e \cos \sigma\right)\left\{\left(\frac{3}{2} p a_{i j}^{2}+e \cos \sigma\right) h-\sqrt{\left.\left(\frac{3}{2} p a_{i j}^{2}+e \cos \sigma\right)^{2}+4\left(\frac{3 \gamma}{4} a_{i j}^{2}+1\right) e^{2} \sin ^{2} \sigma\right\}}\right.}{2 e \sin \sigma \sqrt{\left(\frac{3}{2} p a_{i j}^{2}+e \cos \sigma\right)^{2} h^{2}+4\left(\frac{3 \gamma}{4} a_{i j}^{2}+1\right) e^{2} \sin ^{2} \sigma}}
$$

Since

$$
\frac{3}{2} p a_{i j}^{2}+e \cos \sigma=-e \cos \sigma \mp 2 e \sqrt{\cos ^{2} \sigma-\frac{3}{4}}>0,
$$

we have

$$
\frac{d \omega_{i j}}{d h}<0 \text { if } \sin \sigma>0 \text { and } \frac{d \omega_{i j}}{d h}>0 \text { if } \sin \sigma<0
$$

Thus, when $h$ increases I and $\mathrm{J}$ move to the right (left) if $\sin \sigma>0(<0)$. As $h$ exceeds certain values $h_{i, j}, \mathrm{I}, \mathrm{J}$ change into isolated points.

In Fig. 2, for fixed values $\sigma=\frac{11 \pi}{12} ; \gamma=0.1 ; e=0.06 ; p=0.2$, the resonance curves $1,2,3$ correspond to $h=0 ; 0.005 ; 0.1$

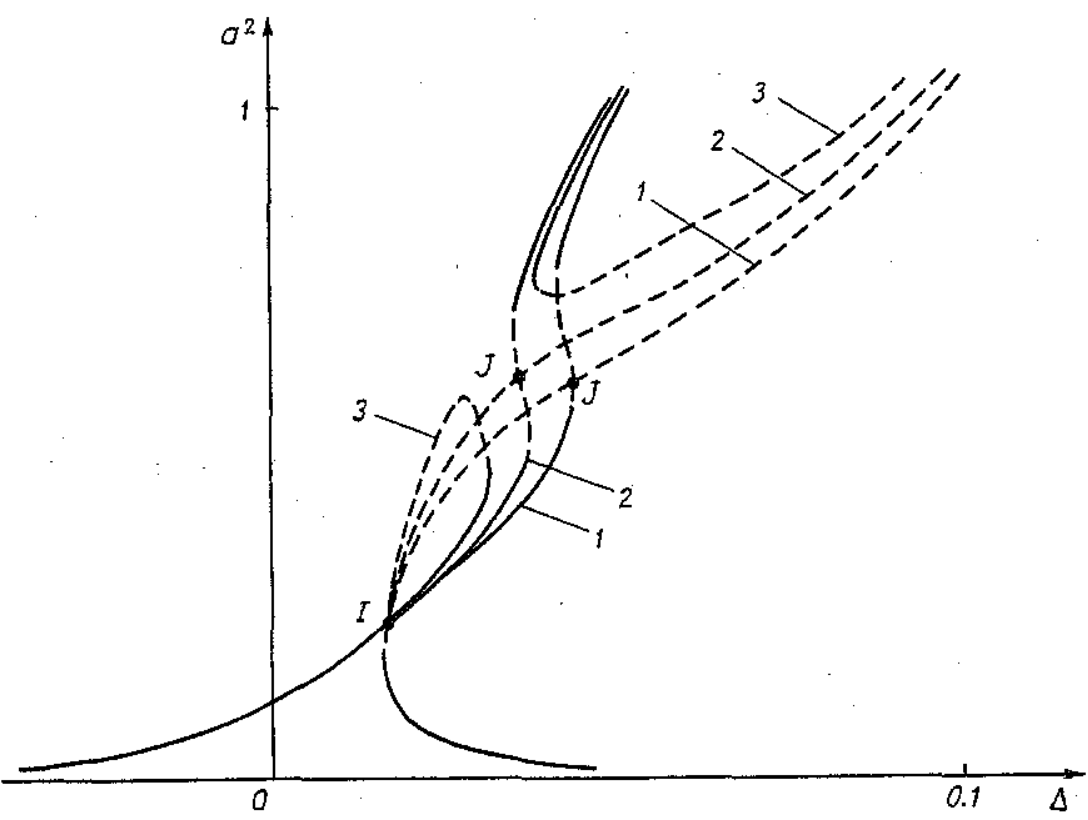

Fig. 2 
In Fig. 3 , for fixed values $\sigma=\frac{13 \pi}{12} ; \gamma=0.1 ; e=0.06 ; p=0.2$, the resonance curves $1,2,3$ correspond to $h=0,0.005 ; 0.1$

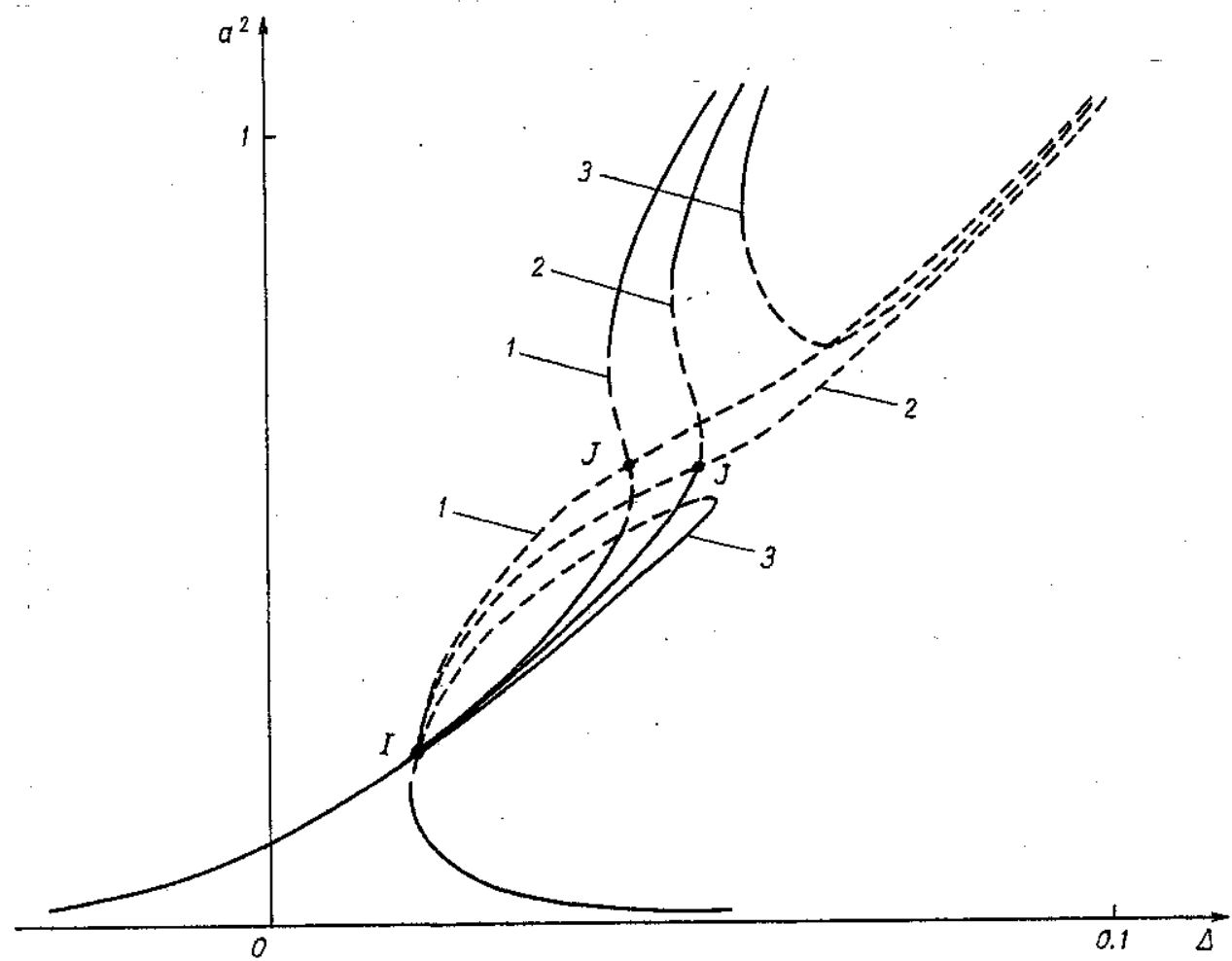

Fig. 8

We see that, increasing $h$ the resonance curves move to the left in the case $\sigma=\frac{11 \pi}{12}$ and to the right in the case $\sigma=\frac{13 \pi}{12}$. As $h$ exceeds certain value, the upper critical representing point $\mathrm{J}$ disappears. The resonance curve is divided into two branches - the upper $C^{\prime}$ of "parabolic" form and the lower $C^{\prime \prime}$ with a loop. Increasing further $h$, the upper branch $C^{t}$ moves up, the lower $C^{\prime \prime}$ moves down, its loop becomes narrower then disappears.

4. System with dephase $0 \leq \sigma<\frac{5 \pi}{6}, \frac{7 \pi}{6}<\sigma<2 \pi$

In this case, the critical region and consequently, critical singular points do not exist. If $h=0$, the resonance curve consists of two branches - the left branch $C^{\prime}$ and the right $C^{\prime \prime}$, located respectively in the left and in the right hand sides of the skeleton curve $\frac{3 \gamma}{4} a^{2}=\Delta$.

Increasing $h, C^{\prime}$ and $C^{\prime \prime}$ approach each other. The two branches $C^{\prime}$ and $C^{\prime \prime}$ may connect togother at an ordinary singular point which disappears immediately and the resonance curve is divided into two branches - the upper and lower ones.

In Fig.4, for fixed values $\sigma=\frac{\pi}{2} ; \gamma=0.1 ; e=0.06 ; p=0.2$, the resonance curve $1,2,3$ correspond to $h=0.04 ; 0.05 ; 0.06$ respectively 


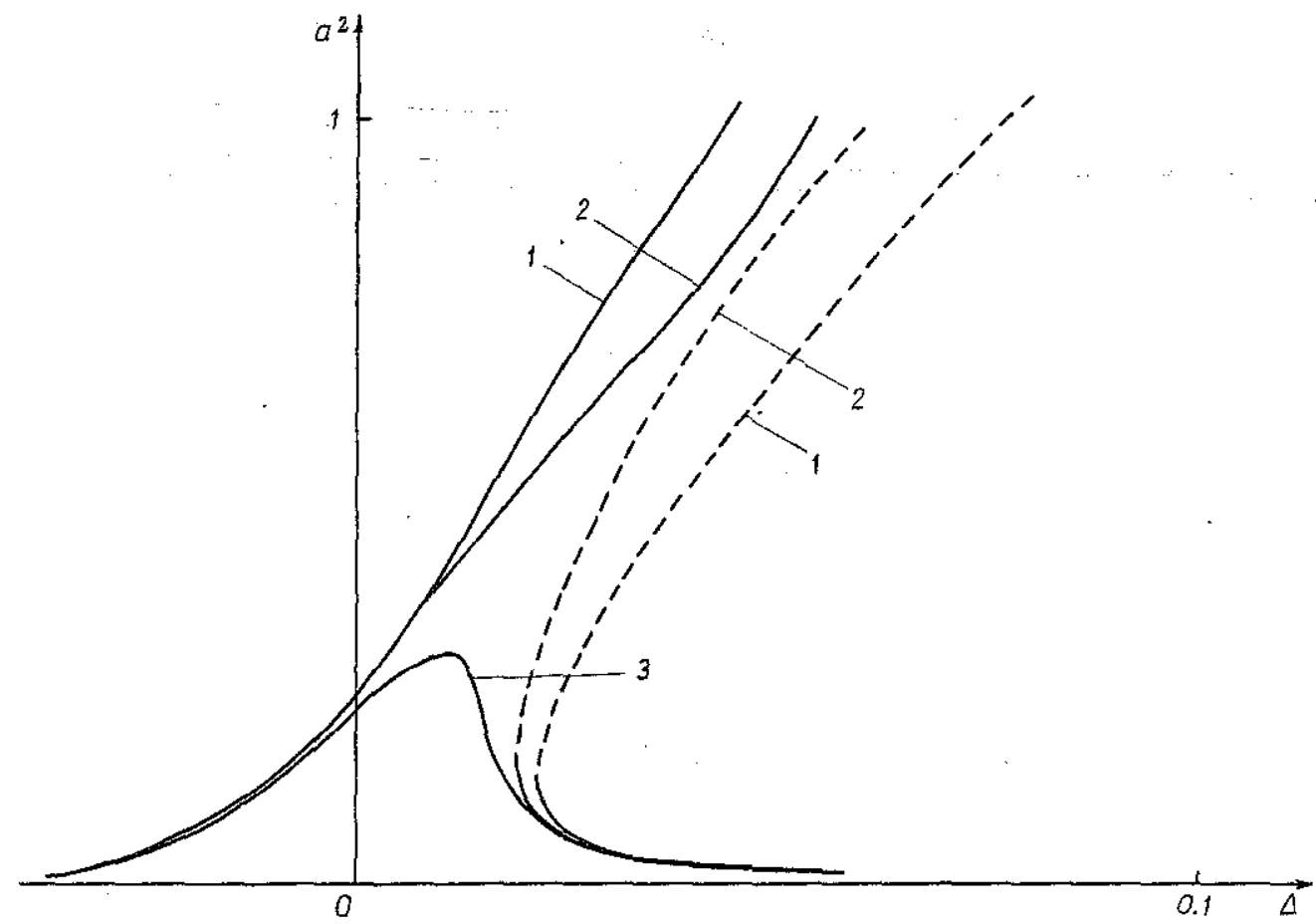

Fig. 4

\section{Stability conditions}

To study the stability character of the stationary oscillations, we use the variational equations:

$$
\begin{gathered}
\delta \dot{a}=-\frac{\varepsilon}{2 \omega} \frac{\partial f}{\partial a} \delta a-\frac{\varepsilon}{2 \omega} \frac{\partial f}{\partial \theta} \delta \theta \\
a \delta \dot{\theta}=-\frac{\varepsilon}{2 \omega} \frac{\partial g}{\partial a} \delta a-\frac{\varepsilon}{2 \omega} \frac{\partial g}{\partial \theta} \delta \theta
\end{gathered}
$$

where $\delta a, \delta \theta$ are small perturbations of $a, \theta$ respectively.

The characteristic equation is of the form:

$$
a \rho^{2}+\frac{\varepsilon}{2 \omega} S_{1} \rho+\frac{\varepsilon^{2}}{4 \omega^{2}} S_{2}=0
$$

and conditions for asymptotic stability are:

$$
\begin{aligned}
& S_{1}=a \frac{\partial f}{\partial a}+\frac{\partial g}{\partial \theta}>0 \\
& S_{2}=\frac{\partial f}{\partial a} \frac{\partial g}{\partial \theta}-\frac{\partial f}{\partial \theta} \frac{\partial g}{\partial a}>0 .
\end{aligned}
$$

The first stability condition is satisfied only for the system with damping:

$$
S_{1}=2 h \omega a>0 \text { i.e. } h>0
$$


For the ordinary part $C_{1}$, the second stability condition can be transformed into the compact form:

$$
\frac{a}{D_{0}} \cdot \frac{\partial W}{\partial a^{2}}>0
$$

and can easily be used to determine ordinary stable portions bounded by vertical tangents.

The stability character of each critical representing nodal point can directly be deduced from that of the ordinary portion considered as containing it. In the figures presented heavy (broken) lines correspond to stable (unstable) oscillations.

\section{Conclusion}

The interaction between parametric and forced oscillations in the fundamental resonance in a quasi-linear oscillating system with time-dependent quadratic non-linearity is examined critical singular points are used to classify different forms of the resonance curve depending on the dephase between two excitations, the resonance curve admits either one or two critical singular points. These points disappear if the damping is strong enough and the resonance curve is divided into two branches - the upper and the lower.

This publication is completed with financial support from the National Basic Research Programme in Natural Sciences

\section{References}

1. Tran Kim Chi. Interaction between parametric and forced oscillations (in Vietnamese) Proceedings of the fifth National Conference on Mechanics T.1 (7-10) Hanoi, 1993.

2. Nguyen Van Dinh. Stationary oscillations in critical cases (in Vietnamese) Journal of Mechanics NCNST of Vietnam T.XVIII, 1996, No 2 (13-19).

Received January \&, 1997

\section{TƯONG TÁC GIŨ̉A KÍCH ĐộNG CUÕ̃NG BỨC VÀ THÔNG SỐ TRONG MộT HỆ DAO ĐộNG Á TUYẾN}

Xét tương tác giữa hai kích động cưỡng bức và thông số trong một hệ dao động á tuyến có phi tuyến bậc hai phụ thuộc thời gian. Điểm kỳ dị tới hạn tương ứng dao động dừng tới hạn được dùng để phân loại dạng các đường cộng hưởng. Phụ thuộc vào độ lệch pha giữa hai kích động, đường cộng hương có một hoặc hai điẻm kỳ dị tới hạn, các điểm này biến mất khi cản đủ mạnh và đường cộng hướng tách thành hai nhánh - nhánh trên và nhánh dưới. 